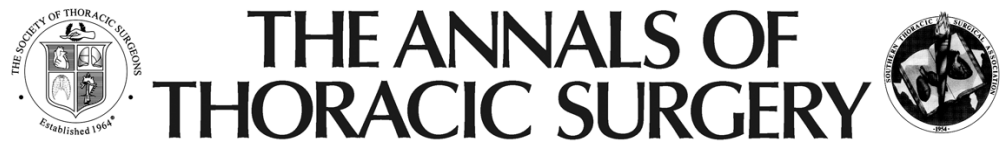

\section{Resuscitation After Prolonged Cardiac Arrest: Role of Cardiopulmonary Bypass and Systemic Hyperkalemia}

Oliver J. Liakopoulos, Bradley S. Allen, Gerald D. Buckberg, Nikola Hristov, Zhongtuo Tan, J. Pablo Villablanca and Georg Trummer Ann Thorac Surg 2010;89:1972-1979

DOI: $10.1016 /$ j.athoracsur.2010.02.052

The online version of this article, along with updated information and services, is located on the World Wide Web at:

http://ats.ctsnetjournals.org/cgi/content/full/89/6/1972

The Annals of Thoracic Surgery is the official journal of The Society of Thoracic Surgeons and the Southern Thoracic Surgical Association. Copyright (C) 2010 by The Society of Thoracic Surgeons. Print ISSN: 0003-4975; eISSN: 1552-6259. 


\title{
Resuscitation After Prolonged Cardiac Arrest: Role of Cardiopulmonary Bypass and Systemic Hyperkalemia
}

\author{
Oliver J. Liakopoulos, MD, Bradley S. Allen, MD, Gerald D. Buckberg, MD, \\ Nikola Hristov, MD, Zhongtuo Tan, MD, J. Pablo Villablanca, MD, and \\ Georg Trummer, MD
}

Department of Surgery, Division of Cardiothoracic Surgery, and Department of Radiological Sciences, David Geffen School of Medicine at University of California Los Angeles, Los Angeles, California, and Department of Cardiothoracic Surgery, Heart Center of the University of Cologne, Germany

Background. The purpose of this study was to determine (1) the role of emergency cardiopulmonary bypass (CPB) after prolonged cardiac arrest and failed cardiopulmonary resuscitation, and (2) the use of systemic hyperkalemia during CPB to convert intractable ventricular fibrillation (VF).

Methods. Thirty-one pigs $(34 \pm 2 \mathrm{~kg})$ underwent 15 minutes of cardiac arrest after induced VF, followed by 10 minutes of cardiopulmonary resuscitation-advanced life support. Peripheral CPB was used if cardiopulmonary resuscitation failed to restore stable circulation. Damage was assessed by evaluating hemodynamics, biochemical variables (creatine kinase-MB, neuron-specific enolase), neurologic deficit score, and brain magnetic resonance imaging.

Results. Cardiopulmonary resuscitation alone was successful in only $19 \%$ (6 of 31 pigs). Cardiopulmonary bypass was initiated in $81 \%$ of animals ( 25 of 31 pigs) either for hypotension (5 of 25 pigs) or intractable VF (20 of 25 pigs). Defibrillation was successful in 7 of 20 animals during the first 10 minutes after initiating CРB.

$S^{u}$ uccessful cardiopulmonary resuscitation (CPR) after cardiac arrest (CA) is possible in approximately $20 \%$ to $40 \%$, yet survival is only $5 \%$ to $10 \%$ and the majority of survivors have permanent brain damage [1]. Conversely, survival rates of up to $50 \%$ can be achieved if CPR with early defibrillation is provided within 4 to 5 minutes, but is negligible if these critical time intervals double, with a greater than $95 \%$ mortality in victims after prolonged (unwitnessed) CA [2-5].

Cardiopulmonary bypass (CPB) expands the time window for successful resuscitation after CA when compared with conventional CPR $[6,7]$, as $80 \%$ to $100 \%$ survival and complete cardiac and neurologic recovery occur after 5 to 10 minutes of CA in dogs $[7,8]$. Time is a critical factor, as mortality increases substantially (approximately 80\%)

Accepted for publication Feb 12, 2010.

Address correspondence to Dr Buckberg, Division of Cardiothoracic Surgery, 62-258 Center for Health Sciences, Los Angeles, CA 90095-1701; e-mail: gbuckberg@mednet.ucla.edu.
Ventricular fibrillation persisted more than $\mathbf{1 0}$ minutes in 13 of 20 pigs, and animals were treated either with repeated defibrillation (6 of 13 pigs) or with a potassium bolus ( 7 of 13 pigs) to induce transient cardiac arrest. Overall survival at 24 hours was $84 \%$ with cardiopulmonary resuscitation (100\% of pigs with hypotension; $71 \%$ in CPB-VF < 10 minutes). Despite CPB, fatal myocardial failure occurred after VF duration of more than $10 \mathrm{~min}$ utes in all pigs treated with electrical defibrillation, whereas hyperkalemia allowed $\mathbf{1 0 0} \%$ cardioversion and $86 \%$ survival. Biochemical variables remained elevated in all groups. Similarly, severe brain injury was present in all animals as confirmed by neurologic deficit score (197 \pm 10$)$ and magnetic resonance imaging.

Conclusions. Emergency CPB after prolonged cardiac arrest improves survival and allows systemic hyperkalemia to convert intractable VF, but fails to reduce neurologic damage.

(Ann Thorac Surg 2010;89:1972-80) (C) 2010 by The Society of Thoracic Surgeons

and no neurologic recovery occurs after CPR-advanced life support (CPR-ALS) after 12.5 minutes of no-flow CA [9]. This is curtailed with $C P B$, with a $70 \%$ survival and $50 \%$ of dogs gaining consciousness after 12.5 minutes of CA. However, after 15 minutes of CA, there is no survival with CPR-ALS and even though CPB allows approximately $25 \%$ to $80 \%$ survival, it fails to prevent severe neurologic damage [7, 10-12]. Furthermore, despite the improvements conferred by $\mathrm{CPB}$, intractable ventricular fibrillation (VF) after prolonged CA remains a survival barrier; it occurs in $20 \%$ to $50 \%$ of animals after CA arrest of 15 minutes, and results in 100\% mortality from cardiogenic shock $[7,11,13]$.

Although these findings imply that emergency CPB is superior to CPR-ALS for resuscitation after prolonged CA, none of these studies implemented a preceding CPR interval that is mandatory in the clinical setting for before CPB is initiated. The present study was aimed to assess whether emergency normothermic $\mathrm{CPB}$ as a resuscita- 
tion tool can improve recovery after 15 minutes of CA (simulating an unwitnessed arrest), when CPR-ALS is ineffective. As a secondary objective, we tested the hypothesis that potassium as a depolarizing cardioplegic agent can offset intractable VF after prolonged CA unresponsive to defibrillation, and improve survival of this otherwise lethal cohort.

\section{Material and Methods}

The study was approved by the Institutional Animal Research Committee of the University of California at Los Angeles. All animals received humane care in compliance with the 1996 NRC Guide for the Care and Use of Laboratory Animals.

Thirty-one Yorkshire-Duroc pigs $(35.2 \pm 1.8 \mathrm{~kg})$ were premedicated with $15 \mathrm{mg} / \mathrm{kg}$ ketamine and $0.5 \mathrm{mg} / \mathrm{kg}$ diazepam intramuscularly. Neuromuscular blockade was achieved by a single pancuronium bolus $(0.2 \mathrm{mg} / \mathrm{kg})$. Endotracheal intubation anesthesia was maintained with inhaled isoflurane $(1.2 \%$ to $1.5 \%)$, using a volume-controlled ventilator (Servo 900C, Siemens Elema, Solna, Sweden) at 12 to 14 breaths/min, 10 to $15 \mathrm{~mL} / \mathrm{kg}$ tidal volume, and adjusted to normalize arterial blood gases.

Heart rate and rhythm were determined by electrocardiography, arterial pressure using a catheter inserted into the left internal thoracic artery, and pulmonary artery pressure by a Swan-Ganz catheter (model 132F5, Baxter Healthcare Corp, Irvine, CA) inserted through the right subclavian vein. Cefazolin (30 mg/kg, GlaxoSmithKline, Philadelphia, PA) was given every 8 hours, and normal saline solution was infused intravenously at 5 to $10 \mathrm{~mL} \cdot \mathrm{kg}^{-1} \cdot \mathrm{h}^{-1}$. Body temperature was kept at $37^{\circ} \mathrm{C}$ using a heating pad. Sterile incisions were performed to expose the right femoral artery and right external jugular vein.

\section{Cardiac Arrest and Resuscitation Protocol}

After baseline measurements, systemic heparinization (300 IU/kg) was given before inducing CA. No-flow CA was maintained for 15 minutes without ventilation after inducing VF with a $60-\mathrm{Hz}(4.5 \mathrm{~A})$ alternating current (AC-Fibrillator, Medtronic, Inc, Minneapolis, MN) stimulus to the right ventricular endocardium using a catheter (Bard Electrophysiology, Lowell, MA) inserted through the right subclavian vein.

The resuscitation protocol was initiated after $15 \mathrm{~min}-$ utes of CA by using the American Heart Association advanced cardiac life support algorithm (ALS) (http:// www.americanheart.org) [14] for 10 minutes, yielding 80 to 100 manual chest compressions per minute and bag ventilation at 12 to 15 breaths/min. Defibrillation was attempted after five cycles of CPR, with $200 \mathrm{~J}$ (Lifepak 9P, Physio-Control Corp, Redmond, WA), and repeated after every five CPR cycles with 300 to $360 \mathrm{~J}$ until spontaneous circulation (ROSC) was restored. Intravenous epinephrine $(0.02$ to $0.04 \mathrm{mg} / \mathrm{kg})$ was administered 2 minutes after beginning CPR, and repeated every 2 to 5 minutes as needed. Lidocaine $(1 \mathrm{mg} / \mathrm{kg})$ was given if a shockable heart rhythm was present, and repeated until reaching the maximal dosage ( $3 \mathrm{mg} / \mathrm{kg}$ ). Cardiopulmonary resuscitation intensity was adjusted to achieve a targeted coronary perfusion pressure of greater than $15 \mathrm{~mm} \mathrm{Hg}$ as indicated by the difference of diastolic arterial pressure and central venous pressure.

Arterial (12F) and venous (19 to $21 \mathrm{~F})$ cannulas (Medtronic, Inc) were inserted into the femoral artery and right atrium (through the external jugular vein) and connected to the CPB circuit during the CPR-ALS interval. Extracorporeal circulation consisted of a membrane oxygenator (Affinity NT 541, Medtronic, Inc), an arterial roller pump (Sarns, Ann Arbor, MI), and a venous centrifugal pump (Biopump BPX-80, Medtronic, Inc) for active venous suction. The extracorporeal circuit was primed with $800 \mathrm{~mL}$ of Plasma-Lyte (Baxter Healthcare Corp), $700 \mathrm{~mL}$ of stored porcine packed donor blood, heparin $(100 \mathrm{IU} / \mathrm{kg})$, and calcium chloride to maintain normocalcemia (approximately $1.2 \mathrm{mmol} / \mathrm{L}$ ).

Restoration of stable circulation (ROSC) during CPRALS was defined as return of supraventricular rhythm with the ability to maintain a mean arterial pressure greater than $50 \mathrm{~mm} \mathrm{Hg}$ for 1 minute. Cardiopulmonary bypass was initiated when 10 minutes of CPR-ALS failed to achieve ROSC, defined as (1) raising mean arterial pressure greater than $50 \mathrm{~mm} \mathrm{Hg}$ (CPB-Hypo), or (2) persistence of ventricular fibrillation (CPB-VF), which was further subdivided into three cohorts based on the length and treatment of VF:

$\mathrm{VF} \leq 10$ min group: Cardioversion was achieved during the first 10 minutes of CPB with countershocks (360 J) repeated every 3 minutes on CPB.

VF $>10$ min group: Animals with intractable VF persisting past the initial 10 minutes on $\mathrm{CPB}$ were randomized to receive either repeated countershocks every 3 to 5 minutes (VF $>10 \mathrm{~min}$ ), or a $15 \mathrm{mEq} / \mathrm{L}$ potassium bolus $(2 \mathrm{mEq} / \mathrm{mL})$ into the arterial line of the CPB circuit for 10 to 20 seconds (VF $>10 \mathrm{~min}+$ potassium).

\section{Cardiopulmonary Bypass Management and Postoperative Care}

Peripheral CPB was established at approximately 300 $\mathrm{mm} \mathrm{Hg}$ arterial partial pressure of oxygen, and flow was adjusted (100 to $150 \mathrm{~mL} \cdot \mathrm{kg}^{-1} \cdot \mathrm{min}^{-1}$ ) to maintain an arterial pressure of 50 to $70 \mathrm{~mm} \mathrm{Hg}$ and a mixed venous oxygen saturation of greater than $70 \%$. Body temperature was kept at $37^{\circ} \mathrm{C}$. Arterial blood gases, electrolytes, and hematocrit were measured every 15 to 30 minutes to ensure optimal levels (Blood Gas System 288, Ciba Corning, Midfield, MA), and activated clotting time was kept greater than 400 seconds. Discontinuation of CPB was initiated when there was a stable heart rhythm for 30 minutes, and mean arterial pressure was greater than 50 $\mathrm{mm} \mathrm{Hg}$ with or without inotropic (dopamine 5 to $10 \mu \mathrm{g}$. $\mathrm{kg}^{-1} \cdot \min ^{-1}$ ) support.

Protamine was given to antagonize systemic heparinization, cannulas were removed, femoral vessels were repaired, and all wounds were closed. Postoperative inotropic support was adjusted to maintain mean arterial pressure between 50 and $70 \mathrm{~mm} \mathrm{Hg}$. Animals were 
extubated when they were able to maintain normal arterial partial pressures of oxygen and carbon dioxide with spontaneous breathing. Animals were monitored for 24 hours after $\mathrm{CPB}$ or for an equal period when $\mathrm{CPB}$ was not used.

\section{Hemodynamic and Biochemical Determinations}

Hemodynamic measurements were made before induction of VF (baseline) and after discontinuation of CPB or at equal time intervals in animals without CPB. Cardiac output was determined by triplicate injections of $4^{\circ} \mathrm{C}$ cold saline solution, and cardiac index and left ventricular stroke work index were calculated using standard equations.

Arterial blood was sampled at baseline, after CPB, and at 24 hours with samples centrifuged $(1,000 \times \mathrm{g})$ for 10 minutes and stored at $-20^{\circ} \mathrm{C}$ until analysis. To mark cardiac and brain injury, creatine kinase-MB activity was assessed by the ultraviolet-spectrophotometric method (Sigma Chemical Co, St. Louis, MO), and neuron-specific enolase was quantified using an Enzyme Immunometric Assay (SynX Pharma Inc, Toronto, ON) according to the manufacturer's recommendation.

\section{Neurologic Deficit Score and Magnetic Resonance Imaging}

Neurologic assessment was performed at 4 and 24 hours after CPB using a species-specific behavior scale, and seizure activity was evaluated $[15,16]$. The neurologic deficit score assesses five neurologic components (cranial nerves, motor and sensory, respiration, consciousness, behavior) with a score of 0 to 100 in each category: $0=$ normal, $500=$ brain death. Neurologic deficit score was independently assessed by 2 laboratory individuals, and the mean value was recorded if scores were different.

After assessment of the 24-hour neurologic deficit score, 4 CPB survivors underwent brain magnetic resonance imaging (MRI) after sedation and endotracheal intubation. Studies were performed on a 3.0-T magnetic resonance system (Magnetom Trio, Siemens Medical Solutions, Malvern, PA), using an axial $\mathrm{T}_{2}$-weighted $\left(\mathrm{T}_{2} \mathrm{~W}\right)$ MRI scan. Tissue hyperintensity on $\mathrm{T}_{2} \mathrm{~W}$ turbo spin echocardiography sequence was used to define completed acute infarction when the diffusion scan was positive. Axial diffusion-weighted MRI was performed to identify sites of acute ischemia when the $T_{2} W$ sequence was normal, or completed infarction when $\mathrm{T}_{2} \mathrm{~W}$ hyperintensity was present. A three-dimensional time of flight magnetic resonance angiogram was used to demonstrate patency of the cerebral vessels, and brain perfusion sequence was obtained using gadodiamide (Omniscan, GE Amersham Health Inc, Princeton, NJ). Scans were evaluated by an experienced neuroradiologist (P.V.).

\section{Statistical Analysis}

Data were expressed as mean \pm standard error of the mean. Two-way analysis of variance with Fisher's least significant difference procedure for post-hoc repeated measurements was used to analyze intragroup and intergroup differences. Perioperative characteristics of groups were analyzed by Student's $t$ test for continuous data and $\chi^{2}$ or Fisher's exact

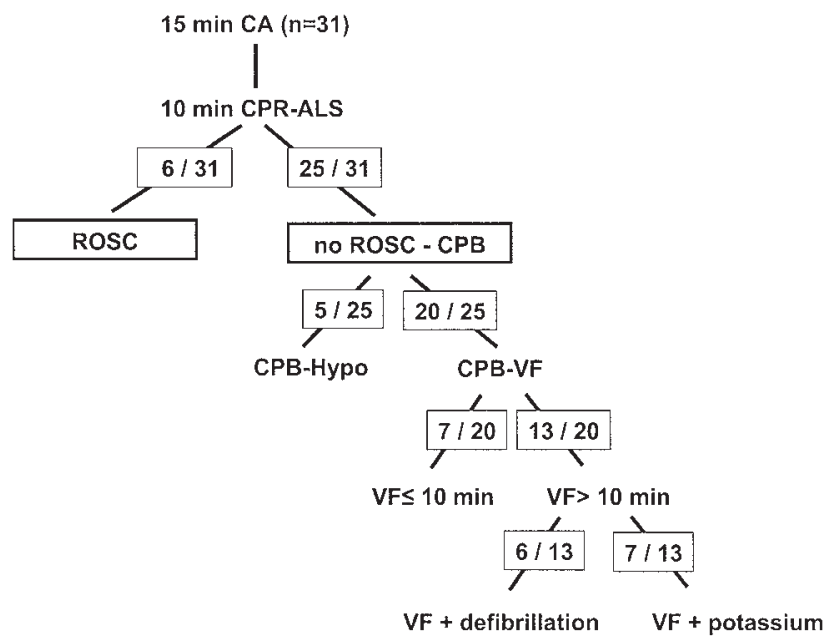

Fig 1. Resuscitation outcome of animals $(n=31)$ subjected to 15 minutes of cardiac arrest (CA) followed by 10 minutes of cardiopulmonary resuscitation-advanced life support (CPR-ALS). Note: CPRALS restored cardiac heart rhythm in 35\% of animals, whereas in $65 \%$ cardiopulmonary bypass (CPB) was established for refractory ventricular fibrillation (VF). (Hypo = hypotension.)

test for categorical data. Probability values less than 0.05 were considered statistically significant.

\section{Results}

Baseline hemodynamic and blood gas data were similar in all animals. The VF amplitude was $0.64 \pm 0.04 \mathrm{mV}$ immediately after VF induction, and declined to $0.33 \pm$ $0.05 \mathrm{mV}(52 \%$ of baseline; $p<0.05)$ at 7.5 minutes, and was almost abolished at 15 minutes $(0.20 \pm 0.03 \mathrm{mV} ; 31 \%$ of baseline; $p<0.05$ ). Cardiopulmonary resuscitationALS consistently produced coronary perfusion pressure greater than $15 \mathrm{~mm} \mathrm{Hg}$ with average coronary perfusion pressure of $29 \pm 3,28 \pm 6$, and $31 \pm 5 \mathrm{~mm} \mathrm{Hg}$ at 3,6 , and 10 minutes of CPR, respectively.

Successful defibrillation was achieved in 11 of 31 animals (35\%) during CPR-ALS, but ROSC was possible in only 6 (19\%; ROSC group; Fig 1). Consequently, $81 \%$ of animals were subjected to emergency $\mathrm{CPB}$ after 10 minutes of CPR-ALS, 5 with persistent hypotension ( $16 \%$; CPB-Hypo) and 20 with intractable VF $(65 \%$; CPB-VF). Seven of $20(35 \%)$ animals with intractable VF were successfully cardioverted during the first 10 minutes of $\mathrm{CPB}(\mathrm{VF} \leq 10 \mathrm{~min})$, whereas 13 of 20 pigs remained in VF more than 10 minutes. Within this cohort, 6 animals underwent repeated defibrillation until successful cardioversion, whereas electromechanical quiescence was quickly achieved in the remaining 7 pigs after delivering potassium (average dose, $14.7 \pm 1.7 \mathrm{mEq} / \mathrm{L}$ ) through the CPB arterial line. Systemic hyperkalemia resulted in a brief interval of asystole (20 to 60 seconds), followed by initial bradycardia, with recovery to a normal heart rate in all but 1 animal that experienced recurrent VF that was defibrillated by one electrical shock.

Total epinephrine dose, number of shocks during CPRALS, time until defibrillation, total CPB time, and inotropic 
Table 1. Perioperative Variables After Cardiac Arrest ${ }^{a}$

\begin{tabular}{|c|c|c|c|c|c|c|c|}
\hline \multirow[b]{2}{*}{ Condition } & \multirow[b]{2}{*}{$\mathrm{N}$} & \multirow{2}{*}{$\begin{array}{l}\text { Epinephrine } \\
(\mu \mathrm{g} / \mathrm{kg})\end{array}$} & \multirow{2}{*}{$\begin{array}{c}\text { Time to Defibrillation } \\
\text { (min) }\end{array}$} & \multicolumn{2}{|c|}{ No. of Shocks } & \multirow{2}{*}{$\begin{array}{l}\text { CPB Time } \\
\text { (min) }\end{array}$} & \multirow{2}{*}{$\begin{array}{l}\text { Dopamine } \\
\quad(\mathrm{n}, \%)\end{array}$} \\
\hline & & & & CPR & СРВ & & \\
\hline ROSC & 6 & $68 \pm 4$ & $6.5 \pm 0.8$ & $4.0 \pm 0.4$ & & & $4(67 \%)$ \\
\hline СРВ-Нуро & 5 & $64 \pm 5$ & $5.4 \pm 1.0$ & $3.8 \pm 0.6$ & & $66 \pm 4$ & $5(100 \%)$ \\
\hline \multicolumn{8}{|l|}{$\mathrm{CPB}-\mathrm{VF}$} \\
\hline $\mathrm{VF} \leq 10 \mathrm{~min}$ & 7 & $77 \pm 3$ & $19.4 \pm 0.4^{\mathrm{b}}$ & $5.1 \pm 0.5$ & $2.1 \pm 0.3$ & $84 \pm 8$ & $5(71 \%)$ \\
\hline $\mathrm{VF}>10 \mathrm{~min}$ & 6 & $74 \pm 2$ & $44.4 \pm 7.8^{\mathrm{b}, \mathrm{c}}$ & $5.7 \pm 0.5$ & $5.5 \pm 0.8^{\mathrm{c}}$ & $155 \pm 19^{\mathrm{b}, \mathrm{c}}$ & $6(100 \%)$ \\
\hline $\mathrm{VF}>10 \mathrm{~min}+$ potassium & 7 & $76 \pm 2$ & $20.1 \pm 0.4^{\mathrm{b}}$ & $5.8 \pm 0.4$ & $1.8 \pm 0.3$ & $99 \pm 9^{b}$ & $4(57 \%)$ \\
\hline
\end{tabular}

${ }^{\mathrm{a}}$ Data are mean \pm standard error of the mean. $\quad{ }^{\mathrm{b}} p<0.05$ compared with the ROSC or CPB-Hypo. $\quad{ }^{\mathrm{c}} p<.05$ compared with VF $\leq 10$ minutes or $\mathrm{VF}>10$ minutes + potassium.

$\mathrm{CPB}=$ cardiopulmonary bypass; $\quad \mathrm{CPR}=$ cardiopulmonary resuscitation; $\quad$ Hypo $=$ hypotension; $\quad$ ROSC $=$ return of spontaneous circulation; $\quad \mathrm{VF}=$ ventricular fibrillation.

support requirements are summarized in Table 1 . Total epinephrine dose and number of defibrillations during CPR were similar in all groups. More total shocks were needed when CPB was initiated for intractable VF and, especially, in the CPB-VF greater than 10 minutes group without potassium, in which $11.1 \pm 0.8$ countershocks were required during CPB $(p<0.05)$, and 1 animal with prolonged VF could not be weaned from CPB owing to cardiac failure. Conversely, all animals in the CPB-Hypo group and 19 of 20 placed on CPB for persistent VF could be weaned from $\mathrm{CPB}$. However, duration on $\mathrm{CPB}$ differed in regard to intractable VF management, with earlier CPB discontinuation being possible in VF less than or equal to 10 minutes and VF greater than 10 minutes plus potassium groups.

\section{Hemodynamic and Biochemical Measurements}

As shown in Table 2, resuscitation on CPB increased heart rate and mean pulmonary artery pressure and decreased left ventricular stroke work index $(p<0.05)$, but did not change cardiac index or mean arterial blood pressure. However, myocardial failure occurred after prolonged VF (CPB-VF $>10 \mathrm{~min}$ ), with reduced mean arterial pressure, cardiac index, and left ventricular stroke work index $(p<$ $0.05)$. These adverse hemodynamic consequences were offset by inducing hyperkalemic arrest with potassium, as CPB duration was lowered to $99 \pm 9$ minutes, fewer shocks $(1.8 \pm 0.3)$ were needed compared with the VF greater than 10 minutes group without potassium (Table 1), and postCPB hemodynamic recovery was similar to all other groups.

Creatine kinase-MB and neuron-specific enolase levels were elevated in all groups after CA (Table 3), implying severe myocardial and brain cellular damage. Biochemical markers of cardiac and cerebral injury tended to be higher after prolonged VF (CPB-VF $>10 \mathrm{~min}$ ), but differences did not reach statistical significance. In the CPB-VF greater than 10 minutes groups, potassium blood

Table 2. Hemodynamic Data ${ }^{a}$

\begin{tabular}{|c|c|c|c|c|c|c|}
\hline Condition & $\begin{array}{c}\mathrm{HR} \\
\text { (beats/min) }\end{array}$ & $\begin{array}{c}\text { MAP } \\
(\mathrm{mm} \mathrm{Hg})\end{array}$ & $\begin{array}{c}\text { MPAP } \\
(\mathrm{mm} \mathrm{Hg})\end{array}$ & $\begin{array}{l}\text { PCWP } \\
(\mathrm{mm} \mathrm{Hg})\end{array}$ & $\left(\mathrm{C} \cdot \min ^{-1} \cdot \mathrm{m}^{-2}\right)$ & $\begin{array}{c}\text { LVSWI } \\
\left(\mathrm{g} \cdot \mathrm{m} / \mathrm{m}^{2}\right)\end{array}$ \\
\hline \multicolumn{7}{|l|}{ Baseline } \\
\hline ROSC & $83 \pm 5$ & $75 \pm 4$ & $15 \pm 1$ & $9 \pm 1$ & $3.4 \pm 0.4$ & $39 \pm 4$ \\
\hline СРВ-Нуро & $94 \pm 9$ & $74 \pm 4$ & $15 \pm 2$ & $9 \pm 1$ & $3.4 \pm 0.2$ & $34 \pm 3$ \\
\hline \multicolumn{7}{|l|}{ CPB-VF } \\
\hline $\mathrm{VF} \leq 10 \mathrm{~min}$ & $90 \pm 4$ & $75 \pm 5$ & $15 \pm 2$ & $9 \pm 1$ & $3.9 \pm 0.5$ & $38 \pm 9$ \\
\hline $\mathrm{VF}>10 \mathrm{~min}$ & $89 \pm 8$ & $75 \pm 3$ & $18 \pm 1$ & $10 \pm 1$ & $3.8 \pm 0.4$ & $37 \pm 3$ \\
\hline $\mathrm{VF}>10 \mathrm{~min}+$ potassium & $94 \pm 4$ & $74 \pm 3$ & $18 \pm 1$ & $11 \pm 1$ & $3.7 \pm 0.3$ & $35 \pm 2$ \\
\hline \multicolumn{7}{|l|}{ After CPB } \\
\hline ROSC & $197 \pm 4^{\mathrm{b}}$ & $75 \pm 4$ & $25 \pm 2^{\mathrm{b}}$ & $9 \pm 1$ & $3.3 \pm 0.4$ & $16 \pm 3^{b}$ \\
\hline СРВ-Нуро & $190 \pm 5^{\mathrm{b}}$ & $69 \pm 6$ & $27 \pm 4^{\mathrm{b}}$ & $10 \pm 2$ & $3.4 \pm 0.4$ & $15 \pm 3^{\mathrm{b}}$ \\
\hline \multicolumn{7}{|l|}{$\mathrm{CPB}-\mathrm{VF}$} \\
\hline $\mathrm{VF} \leq 10 \mathrm{~min}$ & $185 \pm 9^{b}$ & $76 \pm 5$ & $30 \pm 2^{\mathrm{b}}$ & $10 \pm 1$ & $4.1 \pm 0.5$ & $19 \pm 2^{b}$ \\
\hline $\mathrm{VF}>10 \mathrm{~min}$ & $199 \pm 4^{\mathrm{b}}$ & $55 \pm 6^{\mathrm{b}, \mathrm{c}}$ & $28 \pm 2^{\mathrm{b}}$ & $9 \pm 2$ & $2.3 \pm 0.4^{\mathrm{b}, \mathrm{c}}$ & $8 \pm 2^{b, c}$ \\
\hline $\mathrm{VF}>10 \mathrm{~min}+$ potassium & $195 \pm 7^{b}$ & $75 \pm 3$ & $28 \pm 2^{\mathrm{b}}$ & $10 \pm 2$ & $3.8 \pm 0.3$ & $18 \pm 4$ \\
\hline
\end{tabular}

a Data are mean \pm standard error of the mean. $\quad{ }^{\mathrm{b}} p<0.05$ compared with baseline values. $\quad{ }^{\mathrm{c}} p<0.05$ compared with $\mathrm{VF} \leq 10$ min and $\mathrm{VF}>10$ min + potassium.

$\mathrm{CI}=$ cardiac index; $\quad \mathrm{CPB}=$ cardiopulmonary bypass; $\quad \mathrm{HR}=$ heart rate; $\quad$ Hypo = hypotension; $\quad$ LVSWI $=$ left ventricular stroke work index; $\quad \mathrm{MAP}=$ mean arterial pressure; $\quad \mathrm{MPAP}=$ mean pulmonary artery pressure; $\quad \mathrm{PCWP}=$ pulmonary capillary wedge pressure; $\quad \mathrm{ROSC}=$ return of spontaneous circulation; $\quad \mathrm{VF}=$ ventricular fibrillation. 
Table 3. Biochemical Data ${ }^{a}$

\begin{tabular}{|c|c|c|c|c|c|c|}
\hline \multirow[b]{2}{*}{ Condition } & \multicolumn{3}{|c|}{ CK-MB (U/mL) } & \multicolumn{3}{|c|}{ NSE (ng/mL) } \\
\hline & Baseline & After CPB & 24 Hours & Baseline & After CРB & 24 Hours \\
\hline ROSC & $24 \pm 1$ & $120 \pm 13^{b}$ & $113 \pm 20^{\mathrm{b}}$ & $5.6 \pm 0.1$ & $14.4 \pm 0.6^{\mathrm{b}}$ & $16.4 \pm 0.9^{\mathrm{b}}$ \\
\hline СРВ-Нуро & $23 \pm 2$ & $125 \pm 18^{b}$ & $120 \pm 21$ & $5.5 \pm 0.2$ & $15.1 \pm 0.5^{\mathrm{b}}$ & $16.3 \pm 0.7^{\mathrm{b}}$ \\
\hline \multicolumn{7}{|l|}{ CPB-VF } \\
\hline $\mathrm{VF} \leq 10 \mathrm{~min}$ & $24 \pm 1$ & $132 \pm 13^{b}$ & $128 \pm 19^{b}$ & $5.6 \pm 0.1$ & $15.8 \pm 0.6^{\mathrm{b}}$ & $17.2 \pm 0.9^{\mathrm{b}}$ \\
\hline $\mathrm{VF}>10 \mathrm{~min}$ & $27 \pm 2$ & $162 \pm 13^{b}$ & - & $5.4 \pm 0.1$ & $16.5 \pm 0.6^{\mathrm{b}}$ & - \\
\hline $\mathrm{VF}>10 \mathrm{~min}+$ potassium & $25 \pm 1$ & $123 \pm 11^{b}$ & $121 \pm 11^{b}$ & $5.4 \pm 0.2$ & $15.3 \pm 0.7^{\mathrm{b}}$ & $17.0 \pm 1.3^{\mathrm{b}}$ \\
\hline
\end{tabular}

a Biochemical markers of myocardial and neurologic injury were uniformly elevated in all groups after prolonged arrest and CPB and at 24 hours. Note: No biochemical variables could be obtained after prolonged VF on CPB (CPB-VF $>10$ min) owing to $100 \%$ mortality. Data are mean \pm standard error of the mean. ${ }^{b} p<0.05$ compared with baseline values.

$\mathrm{CK}-\mathrm{MB}=\mathrm{MB}$ fraction of creatine kinase; $\quad \mathrm{CPB}=$ cardiopulmonary bypass return of spontaneous circulation; $\quad \mathrm{VF}=$ ventricular fibrillation.

Hypo = hypotension; $\quad$ NSE $=$ neuron-specific enolase; $\quad$ ROSC $=$

concentrations (Fig 2) were higher after potassium bolus injection compared with the no treatment group, with levels returning to normal in both groups by the time $\mathrm{CPB}$ was discontinued.

\section{Survival}

Twenty-four-hour survival was $83 \%$ (5 of 6 pigs) after CPR-ALS and $64 \%$ (16 of 25 pigs) with CPB (Table 4). In the CPB cohort, $100 \%$ of animals survived when hypotension was the reason for CPB. In contrast, 24-hour survival was $71 \%$ (5 of 7 pigs) if VF was successfully converted within 10 minutes (CPB-VF $\leq 10 \mathrm{~min}$ ), whereas mortality was $100 \%$ ( 6 of 6 pigs) if successful defibrillation was not achieved after 10 minutes (VF $>10 \mathrm{~min}$ ). Conversely, 6 of 7 animals survived in the VF greater than 10 minutes plus potassium group, resulting in $86 \%$ survival

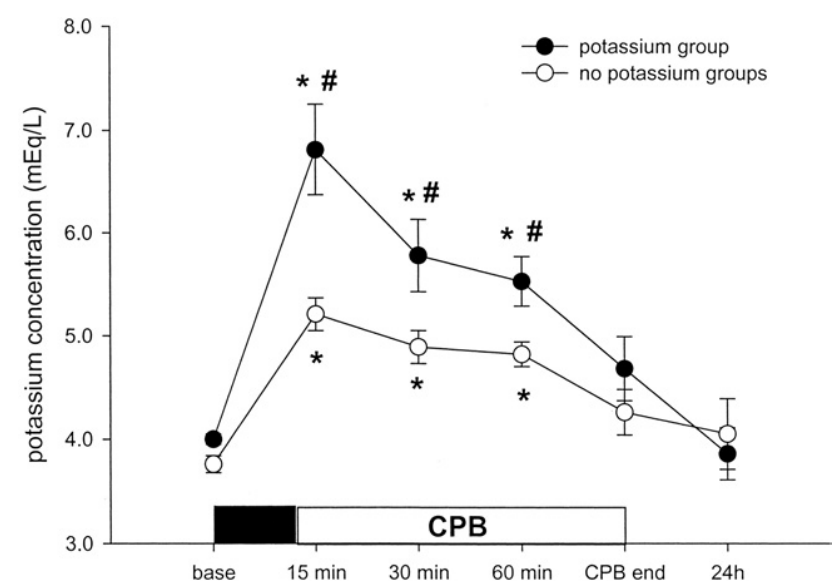

Fig 2. Mean potassium plasma concentrations $(m E q / L)$ in animals with or without receiving a potassium bolus after 10 minutes on cardiopulmonary bypass (CPB). Note the elevated potassium levels after sudden cardiac death and cardiopulmonary resuscitation-advanced life support. Furthermore, animals receiving potassium had higher potassium concentrations at 15 to 60 minutes on cardiopulmonary bypass when compared with untreated animals. Data are mean \pm standard error of the mean. ${ }^{*} p<0.05$ compared with baseline values (base); \#p $<0.05$ compared with untreated animals.
( $p=0.01$ versus VF $>10 \mathrm{~min}$ ). Cardiogenic shock with severe hypotension was responsible for all deaths.

\section{Neurologic Deficit Score and Magnetic Resonance Imaging}

Neurologic deficit score was highest at 4 hours, averaging $220 \pm 9$ for all survivors (Table 4), and improved slightly to $197 \pm 10$ by 24 hours $(p<0.05)$, but did not differ among groups. All animals were conscious and breathing spontaneously but none could sit, stand, walk, eat, or drink. Generalized clonic seizures occurred in $71 \%$ of survivors.

Contrast-enhanced magnetic resonance angiography demonstrated normal, patent cerebral arteries in all animals and no intracranial bleeding, and brain perfusion sequences showed no abnormalities. In the $\mathrm{T}_{1}$-weighted sequence the cerebral cortical mantle, brainstem, and cerebellum were normal with no appreciable mass effect or brain swelling. High $\mathrm{T}_{2} \mathrm{~W}$ signal intensity was present in the basal ganglia, including the globus pallidus (4 of 4 animals), putamen ( 4 of 4 animals), and nucleus caudatus ( 2 of 4 animals), consistent with anoxic or hypoxic brain injury (ie, infarction; Fig 3). Furthermore, the thalamus,

Table 4. Survival, Neurologic Deficit Score, and Seizure Rate $^{a}$

\begin{tabular}{lcccc}
\hline & & \multicolumn{2}{c}{ NDS } & \\
\cline { 3 - 4 } Condition & $\begin{array}{c}\text { Survival } \\
(\mathrm{n} / \%)\end{array}$ & $4 \mathrm{~h}$ & $24 \mathrm{~h}$ & $\begin{array}{c}\text { Seizures } \\
(\mathrm{n} / \%)\end{array}$ \\
\hline ROSC & $5 / 6(83 \%)$ & $224 \pm 20$ & $195 \pm 16$ & $3(60 \%)$ \\
CPB-Hypo & $5 / 5(100 \%)$ & $211 \pm 29$ & $198 \pm 24$ & $3(60 \%)$ \\
$\mathrm{CPB}-\mathrm{VF}$ & & & & \\
$\mathrm{VF} \leq 10 \mathrm{~min}$ & $5 / 7(71 \%)$ & $223 \pm 12$ & $197 \pm 12$ & $4(80 \%)$ \\
$\mathrm{VF}>10 \mathrm{~min}$ & $0 / 6(0 \%)^{\mathrm{b}}$ & - & - & - \\
$\begin{array}{l}\mathrm{VF}>10 \mathrm{~min}+ \\
\quad \text { potassium }\end{array}$ & $6 / 7(86 \%)$ & $219 \pm 16$ & $189 \pm 25$ & $5(83 \%)$ \\
\hline
\end{tabular}

a Data are mean \pm standard error of the mean. $\quad{ }^{\mathrm{b}} p<0.05$ compared with $\mathrm{VF} \leq 10 \mathrm{~min}$ and $\mathrm{VF}>10 \mathrm{~min}+$ potassium.

$\mathrm{CPB}=$ cardiopulmonary bypass; $\quad$ Hypo $=$ hypotension; $\quad$ NDS = neurologic deficit score; ROSC = return of spontaneous circulation; $\quad \mathrm{VF}=$ ventricular fibrillation. 

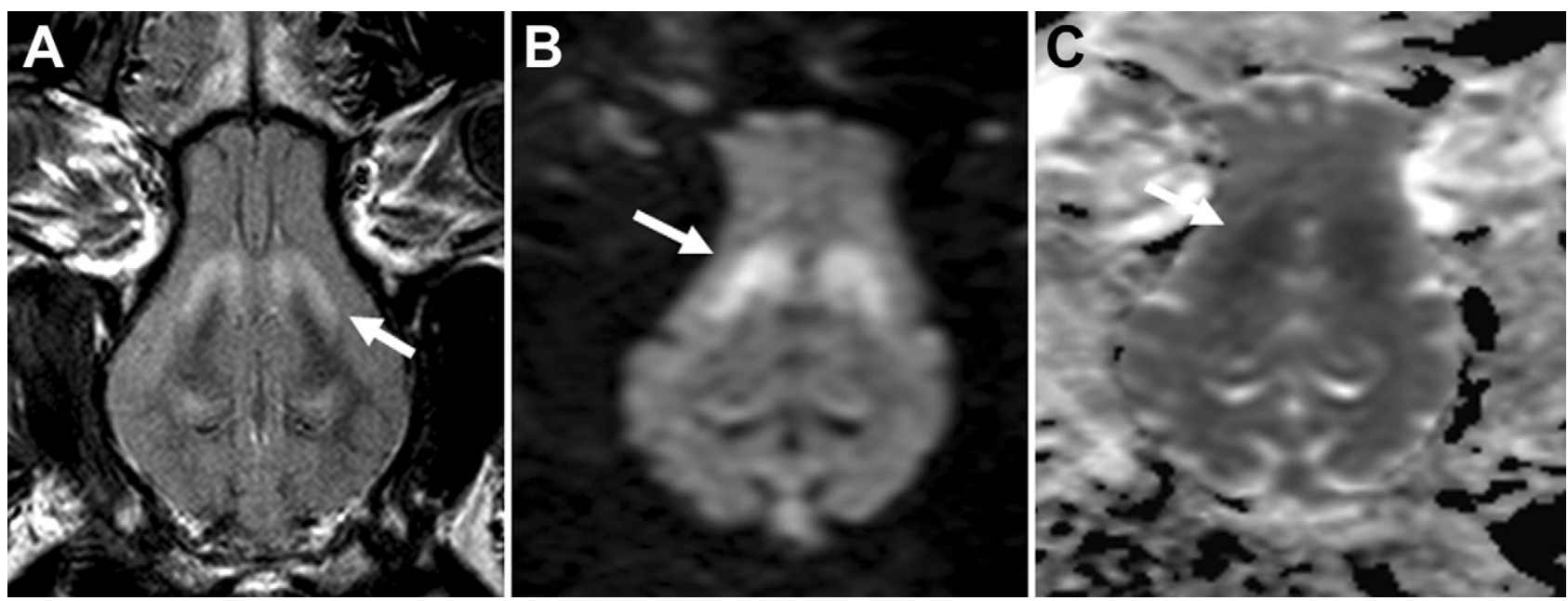

Fig 3. Axial $T_{2}$-weighted slices at the level of the basal ganglia from the same subject. Note the hyperintensity in the caudate, globus pallidus, and putamen in the $T_{2}$ sequence (A) that appears with an increased diffusion signal in the diffusion-weighted image sequence (B), suggesting symmetric hypoxic injury (white arrows). These zones are also accompanied by a hypointensity in the axial sequences (C), indicating true tissue ischemia with completed infarction of the basal ganglia.

cerebellum, and portions of the brainstem were affected in 2 of 4 animals, but none exhibited cortical infarction.

\section{Comment}

Hemodynamic recovery and survival were markedly improved by using emergency $\mathrm{CPB}$ after the otherwise lethal injury of 15 minutes of CA, simulating an unwitnessed CA. Only 19\% successful resuscitation occurred after conventional CPR-ALS, whereas CPB (1) augmented post-CA hypotension, and (2) allowed conversion of intractable VF. Consequently, temporary CPB achieved $64 \%$ survival in animals that would otherwise have succumbed with CPR-ALS alone. Prompt defibrillation is an important element for recovery, as VF greater than 10 minutes resulted in 100\% mortality. Conversely, survival was $71 \%$ when cardioversion was successful during the first 10 minutes on $\mathrm{CPB}$, and $86 \%$ when systemic potassium was used to gain defibrillation on СPB. Unfortunately, improving cardiac survival did not offset the poor neurologic recovery.

Three keynotes to treatment include recognizing the role of $\mathrm{CPB}$ to overcome myocardial stunning after prolonged CA with CPR-ALS [4], using transient hyperkalemia on CBP to convert intractable VF, and insights into improving brain recovery. Studies show that less than 10 minutes of experimental CA followed by normothermic $\mathrm{CPB}$ results in almost complete myocardial and neurologic recovery $[7,8]$. Conversely, after 15 minutes of CA, negligible (approximately 15\%) survival has been reported with CPR-ALS alone [7, 10, 12], and this finding is consistent with the $19 \%$ survival reported in our model. Although CPB allowed $64 \%$ survival after unsuccessful CPR-ALS, it failed to prevent impaired neurologic recovery and thus agrees with previous CPB reports [7, 10-12]. Our model differed from them because 15 minutes of CA was followed by CPR-ALS, a clinically imperative interval to allow the time needed for peripheral cannulation necessary for $\mathrm{CPB}$.

The logic underlying our model of using a period of 15 minutes CA followed by 10 minutes of CPR-ALS relates to (1) the inherent delay in initiating CPR in unwitnessed arrest, or (2) inadequate initial CPR owing to untrained bystanders, as shown in a recent report of $99.4 \%$ mortality, if arrival of a trained resuscitation team was delayed greater than 20 minutes [17]. Although minimal delay in CPR may exist in hospitals with trained teams, implementation of $\mathrm{CPB}$ requires a 10 - to 15 -minute period of CPR-ALS to allow for rapid surgical or percutaneous access of femoral vessels [18, 19]. A recent systematic review of 54 trials emphasizes the value of emergency CPB for treatment of CA [6]. Among 675 patients receiving CPB for CA, $72 \%$ could be weaned successfully off CPB with a $43 \%$ survival to hospital discharge, without reporting neurologic outcomes of survivors [6]. Consistent with this, up to $40 \%$ survival of patients has been reported when $\mathrm{CPB}$ was established within the first 20 to 30 minutes of CPR-ALS [20, 21]. More importantly, simultaneously correcting the underlying cardiac disease that occurs in greater than $60 \%$ of CA patients resulted in an approximately $40 \%$ to $80 \%$ survival with more than $90 \%$ of patients having complete neurologic recovery despite CPR-ALS intervals of longer than 40 minutes [19, 22].

Cardiopulmonary bypass was also a predictor of mortality in our study, as survival was $83 \%$ if animals were subjected to CPB because of persistent hypotension or VF less than 10 minutes. Conversely, $100 \%$ mortality from myocardial dysfunction occurred after prolonged VF. The cardiac consequences of prolonged VF include increased myocardial oxygen consumption, depletion of high-energy phosphates, and calcium overload, resulting in myocardial stunning, ischemic contracture, and necrosis [2]. Further- 
more, CPR and electrical shocks become ineffective with time $[4,23]$ and repeated defibrillation with higher accumulated energy accentuate myocardial injury that worsens post-CA myocardial function and outcome [24]. The high mortality with intractable VF is consistent with previous reports after prolonged CA, where mortality was also $100 \%[11,13]$. Conversely, the $71 \%$ survival after early cardioversion (VF $<10 \mathrm{~min}$ ), or $86 \%$ after potassium depolarization, supports the importance of CPB to maintain perfusion pressure and early treatment to promptly return spontaneous rhythm.

Use of potassium to limit the myocardial causes of mortality after CA borrows from cardiac surgery in which a warm blood cardioplegic reperfusate ("hot shot") is delivered during $\mathrm{CPB}$ [25]. It is also supported by two clinical studies in witnessed CA patients with prolonged VF unresponsive to CPR-ALS, in which CPB was subsequently followed by corrective cardiac surgery using a controlled, substrate-enriched hyperkalemic cardioplegic reperfusion, resulting in excellent post-CA myocardial recovery and approximately $80 \%$ survival $[22,26]$. Thus, systemic potassium administration during CPB initiates a novel treatment option for refractory VF after prolonged CA.

Nonetheless, the capacity to restore satisfactory hemodynamics in otherwise fatal intractable VF using CPB and potassium addresses only the myocardial aspect of survival. Outcomes also depend on avoidance of damage to other organs, especially the brain. Clinical neurologic score marginally improved between 4 and 24 hours, with MRI and neuron-specific enolase confirming severe neurologic damage with lesions in the basal ganglia, thalamus, cerebellum, and brainstem. Similar MRI findings are reported clinically after prolonged CA ( $>6 \mathrm{~min})$, in which increased diffusion-weighted signal intensity occurred in these same brain regions [27]. Hence, CPB as a resuscitative tool for unwitnessed CA successfully salvages the heart, but fails to limit neurologic injury.

Modifying the potassium levels during CPB may reflect only the first step into adjusting the initial reperfusate delivered by the CPB prime to counteract myocardial, brain, and other organ injury. Subsequent alteration of the pump prime and rapid $\mathrm{CPB}$-assisted cooling to moderate whole-body hypothermia $\left(32^{\circ}\right.$ to $\left.34^{\circ} \mathrm{C}\right)$, which has been shown clinically to improve neurologic recovery and reduce mortality in post-CA patients [28], might be the keys to limiting reperfusion organ damage. Our prior studies showed attenuation of brain and whole-body ischemic injury after deep hypothermic circulatory arrest by "controlling" (modifying) the initial CPB reperfusate $[15,16]$. Future studies may use this information to avoid brain injury by adjusting the pump prime to make it a controlled reperfusate (whole-body "hot shot") after prolonged CA.

Limitations of this study include the absence of brain histology to confirm the neurologic damage and the lack of serial MRI and neurologic deficit score examinations for 3 to 7 days to determine the ultimate extent of brain injury. Systemic heparinization before CA, as performed in our study, is not comparable to the clinical situation and might have influenced outcomes. However, Pytte and colleagues [29] demonstrated that pre-CA anticoagulation with enoxaparin $(1 \mathrm{mg} / \mathrm{kg})$ had no influence on hemodynamics or the return of spontaneous circulation after CPR-ALS after 10 minutes of untreated VF. In addition, much larger pre-CA heparin doses (700 IU/kg) were needed to produce favorable effects on survival and neurologic recovery in dogs subjected to 15 minutes of normothermic VF and CPB [30]. Finally, the alternative use of vasopressin and amiodarone (instead of epinephrine and lidocaine), which is equally recommended by the American Heart Association advanced cardiac life support algorithm, may have further improved the ROSC and post-CA outcomes observed in our model.

In conclusion, emergency CPB expands the time window for circulatory resuscitation with improved survival after prolonged CA of 15 minutes, but fails to limit the whole-body or brain reperfusion injury. Furthermore, the treatment option of systemic hyperkalemia for defibrillation allows successful cardioversion of intractable VF with CPB followed by good myocardial recovery and survival. Future therapeutic strategies need to examine ways to limit the deleterious effects of the reperfusionphenomena on post-CA neurologic injury.

This work was supported by a grant from the National Institutes of Health (R01-HL-71729-04).

\section{References}

1. Madl C, Holzer M. Brain function after resuscitation from cardiac arrest. Curr Opin Crit Care 2004;10:213-7.

2. El Menyar AA. The resuscitation outcome: revisit the story of the stony heart. Chest 2005;128:2835-46.

3. Fredriksson M, Herlitz J, Engdahl J. Nineteen years' experience of out-of-hospital cardiac arrest in Gothenburgreported in Utstein style. Resuscitation 2003;58:37-47.

4. Klouche K, Weil MH, Sun S, et al. Evolution of the stone heart after prolonged cardiac arrest. Chest 2002;122:1006-11.

5. Weisfeldt ML, Becker LB. Resuscitation after cardiac arrest: a 3-phase time-sensitive model. JAMA 2002;288:3035-8.

6. Nichol G, Karmy-Jones R, Salerno C, Cantore L, Becker L. Systematic review of percutaneous cardiopulmonary bypass for cardiac arrest or cardiogenic shock states. Resuscitation 2006;70:381-94.

7. Safar P, Abramson NS, Angelos M, et al. Emergency cardiopulmonary bypass for resuscitation from prolonged cardiac arrest. Am J Emerg Med 1990;8:55-67.

8. Levine R, Gorayeb M, Safar P, et al. Cardiopulmonary bypass after cardiac arrest and prolonged closed-chest CPR in dogs. Ann Emerg Med 1987;16:620-7.

9. Pretto E, Safar P, Saito R, Stezoski W, Kelsey S. Cardiopulmonary bypass after prolonged cardiac arrest in dogs. Ann Emerg Med 1987;16:611-9.

10. Ao $\mathrm{H}$, Tanimoto $\mathrm{H}$, Yoshitake A, Moon JK, Terasaki $\mathrm{H}$. Long-term mild hypothermia with extracorporeal lung and heart assist improves survival from prolonged cardiac arrest in dogs. Resuscitation 2001;48:163-74.

11. Kano T, Hashiguchi A, Sadanaga M, et al. Cardiopulmonarycerebral resuscitation by using cardiopulmonary bypass through the femoral vein and artery in dogs. Resuscitation 1993;25:265-281.

12. Yoshitake $\mathrm{A}$, Tanimoto $\mathrm{H}$, Ao $\mathrm{H}$, et al. Does veno-arterial bypass without an artificial lung improve the outcome in 
dogs undergoing cardiac arrest? Resuscitation 2002;54: 159-66.

13. Reich H, Angelos M, Safar P, Sterz F, Leonov Y. Cardiac resuscitability with cardiopulmonary bypass after increasing ventricular fibrillation times in dogs. Ann Emerg Med 1990; 19:887-90.

14. American Heart Association Guidelines for Cardiopulmonary Resuscitation and Emergency Cardiovascular Care: Part 7.2: Management of Cardiac Arrest. Circulation 2005; 112(Suppl):IV-58-66.

15. Allen BS, Veluz JS, Buckberg GD, Aeberhard E, Ignarro LJ. Deep hypothermic circulatory arrest and global reperfusion injury: avoidance by making a pump prime reperfusate-a new concept. J Thorac Cardiovasc Surg 2003;125:625-32.

16. Castella M, Buckberg GD, Tan Z. Neurologic preservation by $\mathrm{Na}^{+}-\mathrm{H}^{+}$exchange inhibition prior to 90 minutes of hypothermic circulatory arrest. Ann Thorac Surg 2005;79:646-54.

17. Herlitz J, Engdahl J, Svensson L, et al. Can we define patients with no chance of survival after out-of-hospital cardiac arrest? Heart 2004;90:1114-8.

18. Hartz R, LoCicero J III, Sanders JH Jr, et al. Clinical experience with portable cardiopulmonary bypass in cardiac arrest patients. Ann Thorac Surg 1990;50:437-41.

19. Sung K, Lee YT, Park PW, et al. Improved survival after cardiac arrest using emergent autopriming percutaneous cardiopulmonary support. Ann Thorac Surg 2006;82:651-6.

20. Hill JG, Bruhn PS, Cohen SE, et al. Emergent applications of cardiopulmonary support: a multiinstitutional experience. Ann Thorac Surg 1992;54:699-704.

21. Overlie PA. Emergency use of cardiopulmonary bypass. J Interv Cardiol 1995;8:239-47.
22. Athanasuleas CL, Buckberg GD, Allen BS, Beyersdorf F, Kirsh MM. Sudden cardiac death: directing the scope of resuscitation towards the heart and brain. Resuscitation 2006;70:44-51.

23. Kolarova J, Ayoub IM, Yi Z, Gazmuri RJ. Optimal timing for electrical defibrillation after prolonged untreated ventricular fibrillation. Crit Care Med 2003;31:2022-8.

24. Gazmuri RJ. Effects of repetitive electrical shocks on postresuscitation myocardial function. Crit Care Med 2000;28: N228-32.

25. Buckberg GD. Update on current techniques of myocardial protection. Ann Thorac Surg 1995;60:805-14.

26. Beyersdorf F, Kirsh M, Buckberg GD, Allen BS. Warm glutamate/aspartate-enriched blood cardioplegic solution for perioperative sudden death. J Thorac Cardiovasc Surg 1992;104:1141-7.

27. Arbelaez A, Castillo M, Mukherji SK. Diffusion-weighted MR imaging of global cerebral anoxia. AJNR Am J Neuroradiol 1999;20:999-1007.

28. The Hypothermia After Cardiac Arrest Study Group. Mild therapeutic hypothermia to improve the neurologic outcome after cardiac arrest. N Engl J Med 2002;346:549-56.

29. Pytte M, Bendz B, Kramer-Johansen J, et al. Prearrest administration of low-molecular-weight heparin in porcine cardiac arrest: hemodynamic effects and resuscitability. Crit Care Med 2008;36:881-6.

30. Ichinose K, Okamoto T, Tashiro M, Tanimoto H, Terasaki H. The effects of pre-arrest heparin administration dose for cardiac arrest model using extracorporeal lung and a heart assist (ECLHA) in dogs. Resuscitation 2006;69:311-8.

\section{INVITED COMMENTARY}

Cardiopulmonary arrest is a leading cause of death in Western society. Cardiopulmonary resuscitationadvanced cardiac life support (CPR-ACLS) is widely thought to be one of the seminal advances in the treatment of heart disease of the 20th century. After nearly 50 years of CPR-ACLS, countless numbers of lives have been saved, and CPR-ACLS is taught in settings ranging from the most advanced quaternary medical centers to local community centers. The benefits are so widely recognized that defibrillators are found in public settings ranging from airports to grocery stores throughout the world. The conundrum is that the benefits of CPR-ACLS are primarily limited to cardiopulmonary arrest that is witnessed, and patients who experience prolonged or unwitnessed cardiac arrest rarely survive.

The report from Liakopoulos and colleagues [1] examines the utility of emergency cardiopulmonary bypass (CPB) in cardiac resuscitation after prolonged cardiac arrest compared with conventional CPR-ACLS. They additionally examined the effect of supplemental hyperkalemic cardiopulmonary arrest in hearts unable to be cardioverted despite being resuscitated on CPB [1].

CPB in this setting is not a new concept. It has been investigated many times before, and CPB is thought to be particularly beneficial for patients who are unable to be cardioverted after CPR-ACLS in the early "electrical phase" of cardiac arrest and are in the later "circulatory" or "metabolic phases" of cardiac arrest [2]. What is unique here is studying CPB after a prolonged period, 15 minutes, of cardiac arrest to simulate the patient who experiences an unwitnessed cardiac arrest. Prolonged periods of cardiac arrest have long been known to reduce the likelihood that CPR-ACLS will be successful [2]. As the authors point out, although successful resuscitation and cardioversion after cardiac arrest can be as high as $40 \%$, survival is generally only $10 \%$ to $15 \%$ due to significant cardiac and brain injury [2]. The longer the downtime, the greater the risk of brain injury and death [3].

In their porcine fibrillation model, CPB improved survival and hyperkalemia-induced asystole improved the chance of defibrillation [1]. The fact that CPB is beneficial is predictable, and the significance of this report is not related to this finding. The utility and practicality of $\mathrm{CPB}$ in this setting is another story and worthy of much discussion. However, what is exciting and a potentially "disruptive concept" is the use of potassium in patients who experience a cardiac arrest. Surgeons use potassium to induce cardiac arrest. We control hyperkalemia postoperatively to treat arrhythmias, even going as far as using dialysis to control it. Will this finding cause us to change our conventional thinking about potassium and ultimately lead to the addition of intravenous potassium to the CPR-ACLS protocols in patients who are unable to be defibrillated early after a cardiac arrest?

Not right now, but perhaps in time. Right now, one has to ask the question: Why does hyperkalemic arrest allow successful defibrillation? It may be that hyperkalemic arrest eliminates electrochemical gradients across the myocardium to allow defibrillation in an environment of uniform electrical quiescence. Whatever the mechanism 


\section{Resuscitation After Prolonged Cardiac Arrest: Role of Cardiopulmonary Bypass and Systemic Hyperkalemia}

Oliver J. Liakopoulos, Bradley S. Allen, Gerald D. Buckberg, Nikola Hristov, Zhongtuo

Tan, J. Pablo Villablanca and Georg Trummer

Ann Thorac Surg 2010;89:1972-1979

DOI: 10.1016/j.athoracsur.2010.02.052

Updated Information

\& Services

References

Citations

Subspecialty Collections

Permissions \& Licensing

Reprints including high-resolution figures, can be found at:

http://ats.ctsnetjournals.org/cgi/content/full/89/6/1972

This article cites 30 articles, 13 of which you can access for free at: http://ats .ctsnetjournals .org/cgi/content/full/89/6/1972\#BIBL

This article has been cited by 1 HighWire-hosted articles: http://ats .ctsnetjournals.org/cgi/content/full/89/6/1972\#otherarticle $\mathrm{s}$

This article, along with others on similar topics, appears in the following collection(s):

Cerebral protection

http://ats.ctsnetjournals.org/cgi/collection/cerebral_protection Extracorporeal circulation

http://ats.ctsnetjournals.org/cgi/collection/extracorporeal_circulatio n

Requests about reproducing this article in parts (figures, tables) or in its entirety should be submitted to:

http://www.us .elsevierhealth.com/Licensing/permissions.jsp or email: healthpermissions@elsevier.com.

For information about ordering reprints, please email: reprints@elsevier.com 